\title{
La competencia científica en los libros de texto de Educación Primaria
}

\author{
Paula Laya Iglesias y Cristina Martínez Losada \\ Facultade de Ciencias da Educación. Universidade da Coruña
}

[Recibido el 12 de febrero de 2019, aceptado el 14 de marzo de 2019]

\begin{abstract}
Este trabajo tiene como objetivo averiguar en qué medida las actividades dirigidas al estudio del ser humano y la salud que proponen los libros de texto LOMCE de 6으 de Educación Primaria, correspondientes a tres editoriales de amplia implantación, promueven la adquisición de la competencia científica de los escolares. El análisis de las actividades (369 en total) se realiza teniendo en cuenta su tipología y el tipo de capacidades que dan ocasión de desarrollar. Respecto a ellas, además de los tres tipos definidos en PISA 2015 se consideran otras capacidades cognitivas presentes en las actividades. Los resultados muestran que la mayoría son cuestiones puntuales de tipo académico, muy centradas en el uso de capacidades ligadas al conocimiento científico en sí, mientras que las directamente asociadas a la competencia científica son poco consideradas. Lo indicado demanda un cambio en los planteamientos de los textos, pues constituyen un eelemento clave en la innovación de la enseñanza
\end{abstract}

Palabras clave: libros de texto; competencia científica; ser humano; educación primaria

\section{Scientific competences in textbooks of Primary Education}

The objective of this work is investigating to what extent the activities addressed to the study of the human being and health, which are proposed in the LOMCE textbooks of 60 of Primary Education, belonging to three editorials of vast establishment, promote the scientific competence acquisition of schoolchildren. The analysis of the activities (369 in total) is developed taking into account its typology and the type of developing capacities. In relation with these capacities, other cognitive capacities present in the activities are taken into account, apart from the three types defined in PISA 2015. The results show that most of them are one-time academic aspects, highly focused on the use of capacities linked to the scientific knowledge in itself, while the ones associated directly to the scientific competence are less considered. The information previously mentioned urges a change in the textbook approach, since they could be a key element in teaching innovation.

Keywords: textbooks, scientific competence; human being; primary education

Para citar el artículo. Laya Iglesias, P. y Martínez Losada, C. (2019). La competencia científica en los libros de texto de Educación Primaria. Ápice. Revista de Educación Científica, 3(1), 71-83. DOI: https://doi.org/10.17979/arec.2019.3.1.5000

Contacto.paulalayaiglesias@gmail.com,cristina.martinez.losada@udc.es 


\section{Introducción}

Siguiendo las directrices europeas, en España las competencias se han incorporado a los últimos currículos de Educación Primaria y Educación Secundaria, entre ellas, aunque bajo distintas denominaciones, se encuentra la competencia científica. Si bien en un principio esta incorporación fue acogida con reticencia (Sanmartí y Sardá, 2007), hoy se reconoce que estas nuevas directrices constituyen un desafío y, a la vez una oportunidad para la mejora de la enseñanza de las Ciencias (Vilches y Gil, 2010), en cuanto demandan la superación de planteamientos tradicionales ampliamente criticados. En este sentido cabe destacar que, a pesar de que la importancia de los procedimientos y habilidades en el aprendizaje científico ha sido ampliamente justificada desde un punto de vista teórico, adquiriendo a partir de la LOGSE la categoría de contenidos a enseñar, estudios realizados muestran que tal importancia no se ha trasladado a la realidad de las aulas (Martínez Losada y García Barros, 2003). En este sentido se ha podido constatar que las actividades habitualmente empleadas eran excesivamente reiterativas y dejaban poco espacio al desarrollo de procedimientos de carácter investigativo relacionados con la indagación y la resolución de problemas, siendo las exigencias relativas a la comunicación muy reducidas. De esta forma, la enseñanza ha seguido priorizando la adquisición de conocimientos teóricos declarativos y descontextualizados, con el consiguiente incremento en la insatisfacción e interés de los jóvenes hacia la Ciencia y su aprendizaje (Rocard, 2007).

Por otra parte, el marco competencial actual implica no solo que el alumnado vaya desarrollando diferentes procedimientos y habilidades, sino también que sea capaz de aplicarlos en contextos y situaciones variadas de la vida (Pro, 2013).

En cualquier caso, la indefinición del concepto de competencia científica en los currículos, hace que su utilización como referente para el desarrollo de la enseñanza y para la elaboración o elección de los materiales didácticos resulte complejo (Rodríguez, Pro y Molina, 2018), con lo cual se corre el riesgo de que esta nueva reforma curricular se quede, como ya ocurrió en el pasado (González Rodríguez, Martínez Losada y García Barros, 2009), en una mera declaración de intenciones. En este sentido, no se puede olvidar que, a pesar de las críticas recibidas (Del Carmen, 2001), los libros de texto son el material comúnmente empleado por el profesorado como guía y apoyo de sus decisiones docentes respecto a qué contenidos tratar, cómo tratarlos, qué cuestiones plantear, etc. (Occelli y Valeiras, 2013), desempeñando, por tanto, un referente básico en el desarrollo curricular y, por extensión un papel esencial en el aprendizaje del alumnado. De hecho, existe una línea de investigación específica centrada en las características de los textos escolares, realizándose estudios desde diferentes perspectivas: el tipo de ilustraciones que incluyen (López y Postigo 2014), los contenidos que tratan (Rodríguez, de las Heras, Romero, y Cañal, 2014), etc.

De acuerdo con lo indicado, en el momento actual resulta relevante analizar en qué medida los textos escolares han asumido las nuevas tendencias curriculares. Más concretamente y dado que la competencia científica se demuestra en la acción (Sanmartí y Márquez, 2017), es decir, en un "saber hacer", el análisis deberá centrarse en las características de las actividades que proponen.

\section{La competencia científica como referente curricular}

La competencia científica se ha considerado un eje central en la educación científica de los estudiantes, (Kauertz, Newman y Haertig, 2012), destacándose que su desarrollo es clave en la formación de los jóvenes, que deberán enfrentarse a situaciones y problemas del mundo globalizado y tecnológicamente avanzado actual en el que viven (COSCE, 2011; Fensham, 2009; Blanco-López, España-Ramos, González-García y Franco-Mariscal, 2015). 
A lo largo de los años se han dado varias definiciones de competencia científica (Gutiérrez, 2006; Cañas, Martín-Díaz y Nieda, 2007), al tiempo que el propio concepto ha ido evolucionando (Sanmartí y Márquez, 2017). En concreto el informe PISA 2015 (MECD, 2016) la define como "la capacidad de interesarse e implicarse en temas científicos e ideas sobre la ciencia como ciudadano consciente y reflexivo". Además identifica tres sub-competencias asociadas a la misma: a) explicar fenómenos científicamente; b) evaluar y diseñar la investigación científica; c) interpretar datos y pruebas científicas. Indudablemente se trata de una capacidad compleja, que implica diferentes tipos de conocimientos, habilidades y actitudes, que han de movilizarse conjuntamente para lograr una acción eficaz (OECD, 2002).

El interés creciente por el enfoque competencial y la necesidad de concretar su significado, han dado lugar a diversos trabajos que tratan de identificar los tipos de capacidades que deben desarrollarse prioritariamente en las aulas. Concretamente Pedrinaci (2012) distingue entre las relativas al conocimiento de la Ciencia, a la práctica de la Ciencia y a la naturaleza de la Ciencia y sus relaciones con la tecnología y la sociedad. A su vez, Cañal $(2011 ; 2012)$ identifica cuatro dimensiones de la competencia científica, que relaciona con diferentes tipos de aprendizajes o saberes básicos: a) conceptual, relativa a la comprensión de los principales conceptos y modelos científico-escolares y ser capaz de usarlos para describir, explicar o predecir cosas o fenómenos de la realidad cotidiana; b) metodológica, relacionada con el conocimiento y uso de procesos necesarios para abordar el estudio de los fenómenos desde una perspectiva científica, la búsqueda y selección de información relevante y la producción y valoración de conclusiones y argumentaciones; c) actitudinal, asociada a la valoración e interés por el conocimiento, la investigación y resolución de problemas científicos y la toma de decisiones; y d) integrada, que implica el establecimiento de relaciones entre distintas capacidades científicas.

De acuerdo con lo indicado, para promover el desarrollo de la competencia científica será necesario que las actividades de aula proporcionen oportunidades para que los estudiantes modelicen con objeto de generar explicaciones válidas, investiguen para generar nuevas ideas en respuesta a preguntas o problemas y puedan argumentar y evaluar la validez de las mismas (Osborne, 2014; Romero-Ariza, 2017).

Todas estas capacidades deberán plantearse en relación con unos determinados conocimientos, con un contexto y con el paralelo desarrollo de actitudes (Cañas y otros, 2007). Concretamente para la Educación Primaria esos conocimientos giran en torno al ser humano y la salud, los seres vivos, los materiales y la energía. Respecto a cada uno de ellos resulta imprescindible la búsqueda contextos del mundo real que resulten relevantes personal y socialmente al ciudadano (Cañas y Martín-Díaz, 2010; Blanco y España, 2012). En esta línea, se ha señalado el interés del tratamiento de situaciones y problemas de la vida cotidiana, que resulten próximos y asequibles al alumnado (Gilbert, 2006; Sanmartí, Burgoa y Nuño, 2011), así como el uso de problemas o controversias de tipo socio-científico, relacionados, por ejemplo, con el consumo, la salud o el medio ambiente (Díaz-Moreno y Jiménez Liso, 2012). A su vez, el énfasis en la utilidad del conocimiento para la vida y los enfoques contextualizados contribuirá a fomentar actitudes de interés por la Ciencia en cuanto permiten dar respuesta a los problemas del mundo, al tiempo que promueven el desarrollo del pensamiento crítico del alumnado (Blanco López, España Ramos y Franco-Mariscal, 2017) y, con ello, la capacidad de dar opiniones argumentadas, posicionarse y tomar decisiones en contextos personales y sociales variados.

\section{Objetivos}

Este trabajo tiene como objetivo averiguar en qué medida las actividades que proponen los libros de texto LOMCE de Educación Primaria más utilizados por el profesorado de la 
Comunidad Autónoma de Galicia promueven la adquisición de la competencia científica de los escolares.

Con objeto de acotar el estudio se eligió el último curso de Primaria y un bloque temático concreto, "El ser humano y la salud". Por otra parte, aunque la competencia científica requiere también de unos conocimientos y actitudes, el trabajo se centra específicamente las capacidades implicadas en la misma. Más concretamente, con este estudio se pretende:

- Analizar la tipología de las actividades que proponen los textos.

- Identificar y valorar el tipo de capacidades que dan ocasión de desarrollar las actividades propuestas.

\section{Metodología}

Para este estudio se utilizaron los textos LOMCE correspondientes a tres editoriales de amplia implantación en Educación Primaria (que, en adelante, denominaremos como A, B y C). En concreto se analizaron las actividades que proponen los libros de texto dirigidos a 60 curso. En ese curso, respecto al bloque temático elegido, las tres editoriales trabajan en dos o tres unidades didácticas diferentes aspectos relacionados con dos funciones del ser humano, la nutrición y la reproducción. Ninguna de ellas incluye en ese curso como objeto de estudio la función de relación. El número total de actividades analizadas fue 369 de las que 86 corresponden a la editorial A, 139 a la editorial B y 144 a la editorial C.

El análisis de las actividades se realizó teniendo en cuenta su tipología y su contribución al desarrollo de la competencia científica. En cada caso se elaboraron dossiers en el que se contemplan diferentes dimensiones y unidades de análisis.

Respecto a la tipología de las actividades se consideró: a) el tipo de actividad, es decir, si se trata de una cuestión puntual o una actividad más amplia, formada por varias cuestiones o apartados interrelacionados entre sí; b) su situación en la unidad didáctica, al inicio, de forma intercalada a lo largo del texto o al final de la misma; c) la incorporación de recursos específicos necesarios para resolverla, como por ejemplo, dibujos o esquemas, objetos reales, uso de diccionarios, videos, acceso a internet, etc. y d) su grado de contextualización, donde se considera si la actividad hace referencia a un contexto cotidiano, de salud (personal o social) y si es de tipo académico.

Para el análisis de las capacidades se ha tomado como referente las dimensiones de la competencia científica señaladas en distintos trabajos (Cañas y otros, 2007; Cañal, 2011; 2012; Pedrinaci, 2012; MECD, 2016). Además, hemos considerado conveniente tener en cuenta otro tipo de capacidades, que denominamos de tipo cognitivo básico, directamente relacionadas con la identificación y procesado del conocimiento científico en sí. Esta decisión se tomó debido a que una primera revisión de las actividades que plantean los textos muestra que muchas de ellas inciden específicamente en el desarrollo de este tipo de capacidades. Por otra parte, desde la perspectiva competencial actual la adquisición de conocimientos sigue resultando relevante, en la medida que el alumnado tendrá que emplear el conocimiento científico disponible para resolver situaciones y problemas de la vida.

De acuerdo con todo ello se han delimitado cuatro tipos de categorías estableciéndose en cada una de ellas las correspondientes subcategorías:

a) Capacidades tipo I, ligadas al conocimiento científico en sí -identificar características, establecer relaciones, comparar y definir o conceptualizar qué es-;

b) Capacidades tipo II, relativas al uso del conocimiento en situaciones concretas-describir qué pasa, explicar cómo sucede y justificar teóricamente por qué sucede-; 
c) Capacidades tipo III, correspondientes a procesos científicos necesarios para abordar el estudio de situaciones -observar, buscar información en diferentes fuentes, plantear hipótesis y diseñar estrategias, realizar experiencias para obtener datos-;

d) Capacidades tipo IV, asociadas al uso de pruebas científicas -analizar datos, obtener conclusiones basadas en pruebas, argumentar a favor o en contra de las mismas-.

En la tabla 1 se muestran ejemplos representativos de actividades en las que se trabaja cada capacidad.

Tabla 1. Tipos de capacidades, capacidades concretas de cada tipo y ejemplos representativos de actividades

\begin{tabular}{|c|c|c|}
\hline \multicolumn{2}{|c|}{ Capacidades } & Ejemplos representativos de actividades \\
\hline \multirow{4}{*}{ 을 } & $\begin{array}{l}\text { Identificar } \\
\text { caracterís- } \\
\text { ticas }\end{array}$ & $\begin{array}{l}\text { - Indica cuales de las siguientes sustancias transportan los glóbulos rojos en } \\
\text { nuestra sangre: agua, oxígeno, dióxido de carbono, nutrientes. } \\
\text { - Escribe cinco hábitos saludables que realices en tu vida cotidiana. }\end{array}$ \\
\hline & $\begin{array}{l}\text { Establecer } \\
\text { relaciones }\end{array}$ & $\begin{array}{l}\text { - Haz un esquema en el que relaciones las partes del aparato reproductor mas- } \\
\text { culino y su función. } \\
\text { - Relaciona los tres órganos anteriores (....) con los productos de desecho que } \\
\text { expulsa cada uno de ellos. }\end{array}$ \\
\hline & Comparar & $\begin{array}{l}\text { - Escribe qué diferencias hay entre el aire que entra en los pulmones y el que } \\
\text { sale de ellos. } \\
\text { - Compara las dos dietas que se muestran a continuación. }\end{array}$ \\
\hline & Definir & - ¿Qué es el plasma? Define: a) digestión; b) absorción ... \\
\hline \multirow{3}{*}{$\begin{array}{l}= \\
\stackrel{2}{2} \\
i=\end{array}$} & Describir & $\begin{array}{l}\text { - Escribe el recorrido que siguen los óvulos y los espermatozoides desde que } \\
\text { se producen hasta que salen al exterior cuando no hay fecundación. } \\
\text { - Describe qué les ocurre a los alimentos durante la digestión. }\end{array}$ \\
\hline & Explicar & $\begin{array}{l}\text { - Explica la relación que existe entre el desarrollo del feto, el parto y la forma- } \\
\text { ción del ombligo. } \\
\text { - Explica para qué utilizamos las proteínas que ingerimos }\end{array}$ \\
\hline & Justificar & $\begin{array}{l}\text { - Por qué las glándulas sudoríparas intervienen en la función de excreción. } \\
\text { - Por qué si pongo un filete en la mano las células de mi piel no pueden nutrir- } \\
\text { se con él? ¿Qué debe ocurrir para que puedan aprovecharlo? }\end{array}$ \\
\hline \multirow{4}{*}{$\begin{array}{l}\equiv \\
\stackrel{2}{\circ} \\
i=\end{array}$} & Observar & $\begin{array}{l}\text { - Fíjate en los dibujos e indica... } \\
\text { - Observa la preparación (células sanguíneas) en un microscopio.... }\end{array}$ \\
\hline & $\begin{array}{l}\text { Buscar in- } \\
\text { formación }\end{array}$ & $\begin{array}{l}\text { - Pídeles a tus padres tu cartilla de vacunación y analízala. } \\
\text { - Busca información en internet sobre... }\end{array}$ \\
\hline & $\begin{array}{l}\text { Proponer } \\
\text { hipótesis/ } \\
\text { estrategias }\end{array}$ & $\begin{array}{l}\text { - Por equipos escoged uno de los siguientes temas... después planificad un tra- } \\
\text { bajo de investigación. ¿De dónde vais a obtener la información? ¿Qué pregun- } \\
\text { taréis? ¿A quién? }\end{array}$ \\
\hline & $\begin{array}{l}\text { Realizar ex- } \\
\text { periencias }\end{array}$ & $\begin{array}{l}\text { - Vamos a realizar un experimento para comprobar que el corazón funciona } \\
\text { más rápidamente cuando hacemos ejercicio que cuando estamos en reposo... } \\
\text { Un compañero manejará un reloj o cronómetro y el otro le tomará el pulso.... }\end{array}$ \\
\hline \multirow{3}{*}{$\begin{array}{l}\geq \\
\stackrel{2}{\circ} \\
i=\end{array}$} & $\begin{array}{l}\text { Analizar una } \\
\text { información }\end{array}$ & $\begin{array}{l}\text { - Fíjate en las gráficas... ¿En qué tipo de actividad gastamos más energía? Ra- } \\
\text { zona la respuesta. }\end{array}$ \\
\hline & $\begin{array}{l}\text { Elaborar } \\
\text { conclusions }\end{array}$ & $\begin{array}{l}\text { - Con este experimento pudiste simular una caja torácica humana ... Escribe un } \\
\text { breve informe explicando si la simulación te parece adecuada o si, por el contra- } \\
\text { rio, cambiarías algo tu modelo. }\end{array}$ \\
\hline & Argumentar & $\begin{array}{l}\text { - Diseña un cartel para promover entre los escolares la dieta sana. } \\
\text { - Escribe } 3 \text { razones que darías a un fumador para que abandonase ese hábito. }\end{array}$ \\
\hline
\end{tabular}




\section{Resultados}

\section{Tipología de las actividades}

Más del $80 \%$ de las actividades que proponen los libros de texto de las tres editoriales son cuestiones puntuales relacionadas con los contenidos que tratan de forma expositiva (Figura 1). Sin embargo, las editoriales también proponen actividades más amplias que incluyen diferentes cuestiones o apartados sobre un aspecto concreto o una situación relacionada con el tema objeto de estudio (entre el $10.1 \%$ y el $16.0 \%$ del total que propone cada editorial).

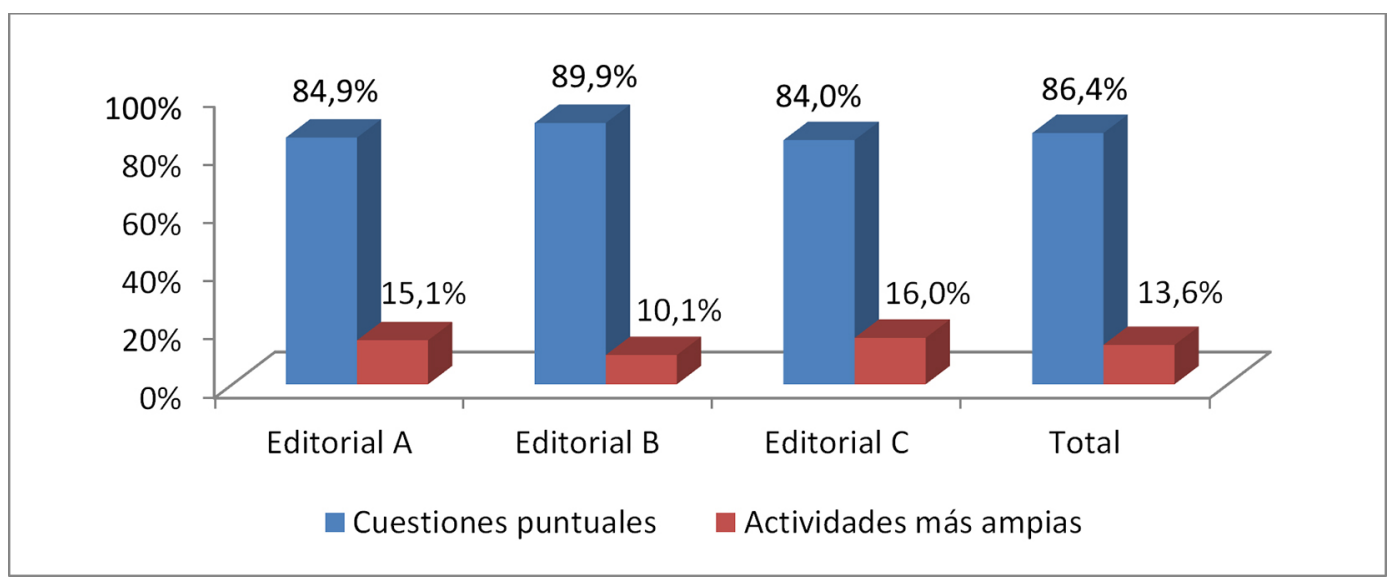

Figura 1. Proporción de cuestiones puntuales y de actividades más amplias que incluyen las editoriales

En general, las editoriales sitúan la mayoría de las actividades de forma intercalada o bien al final de cada unidad didáctica (Figura 2). Así, salvo excepciones, las cuestiones puntuales se sitúan siempre intercaladas o al final del texto ( $42.6 \%$ y $54.5 \%$, respectivamente). Sin embargo, las actividades más amplias se sitúan al final (56.0\%) y, en menor medida, intercaladas o al inicio del tema (26.0\% y el $18.0 \%)$. En concreto la editorial A sitúa las cuestiones sobre todo de forma intercalada (61.6\%), la editorial B las sitúa en mayor medida al final $(57.1 \%$ ) y la C las sitúa tanto de forma intercalada como al final (44.6\% y $51.2 \%$ ). Asimismo, mientras que esta última sitúa las actividades más amplias preferentemente al final (82.60\%), la editorial B las sitúa en mayor medida de forma intercalada (57.1\% frente al $21.4 \%$ iniciales y finales, respectivamente) y la editorial A lo hace de una manera más equilibrada $(30.8 \%, 23.1 \%$ y $46,2 \%)$.

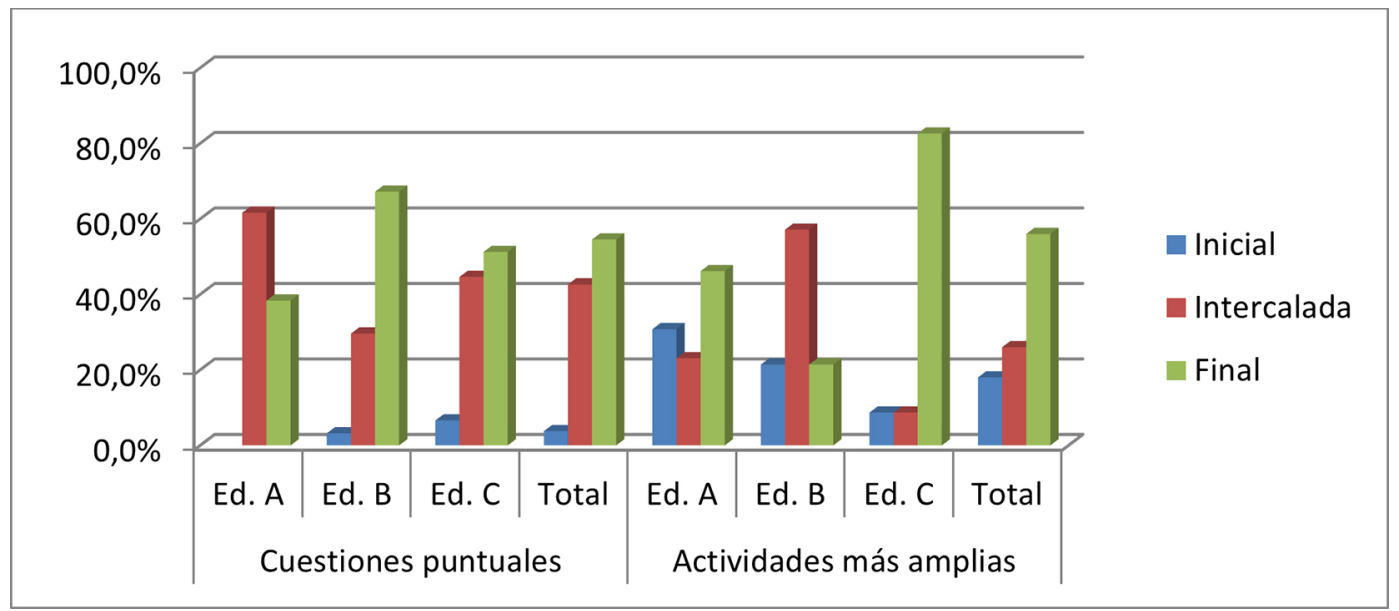

Figura 2. Distribución de las actividades en función de su situación en la unidad didáctica 
Por otra parte, el $70 \%$ de las actividades más amplias requieren el uso de algún tipo de recurso específico de obtención de información, mientras que esto ocurre en menos del $25 \%$ del total de cuestiones puntuales que proponen (Figura 3). Sin embargo, cabe señalar que, el tipo de recurso mayoritariamente empleado son imágenes, esquemas o pequeñas lecturas relacionados con la cuestión o cuestiones planteadas. Por el contrario, por ejemplo, la búsqueda de información en distintas fuentes (internet, otros materiales escritos, otras personas...) es muy poco frecuente.

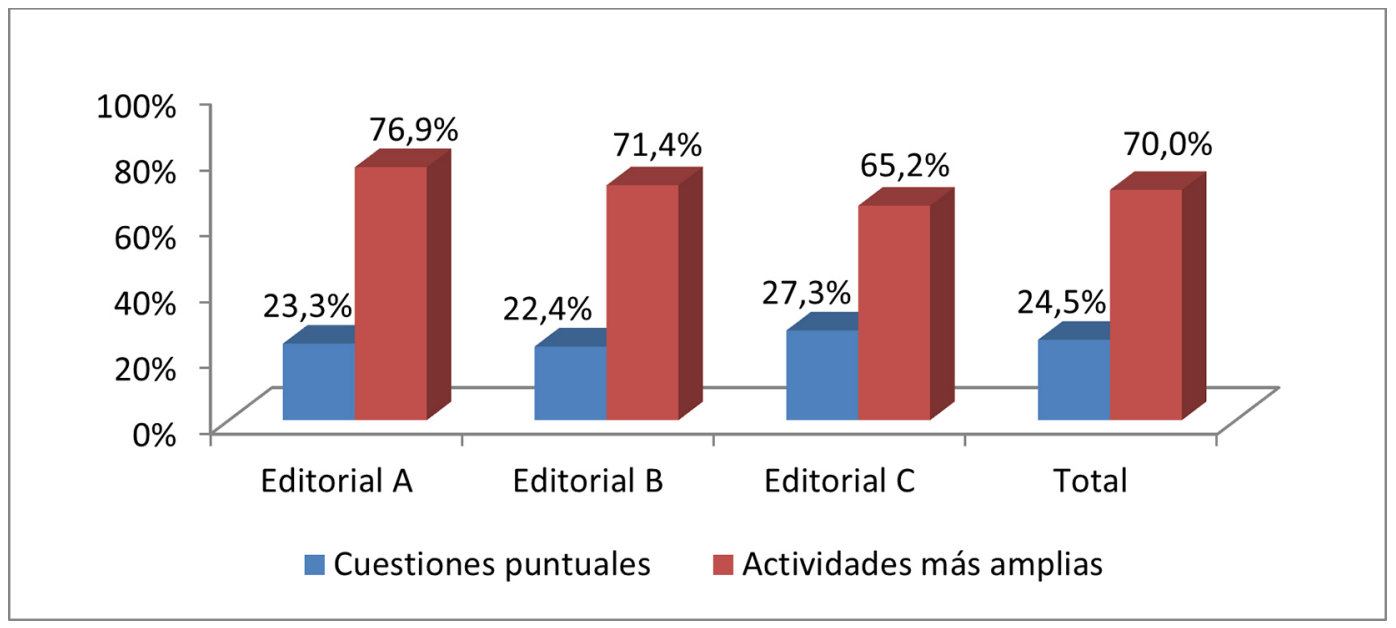

Figura 3. Proporción de actividades que implican el uso de recursos específicos

Con relación al contexto también se aprecian diferencias según el tipo de actividad (Figura 4). Así, las editoriales, plantean la mayoría de las cuestiones puntuales en un contexto académico, es decir, relacionado con el contenido teórico del tema objeto de estudio, siendo el porcentaje de las mismas especialmente alto en el caso de la editorial A ( $86.3 \%$ frente al $78.7 \%$ y $67.5 \%$ de las editoriales B y C).

Sin embargo, las actividades más amplias se enmarcan en mayor medida en un contexto cotidiano y/o de salud. En concreto las editoriales A y B las contextualizan en mayor medida en situaciones cercanas y conocidas de los escolares $(53.8 \%$ y $50.0 \%$ ) que en un tema o problema de salud personal o social $(23.1 \%$ y $21.4 \%$. Por el contrario la editorial C las enmarca de forma más equilibrada en ambos contextos (30.0\% y $47.8 \%)$.

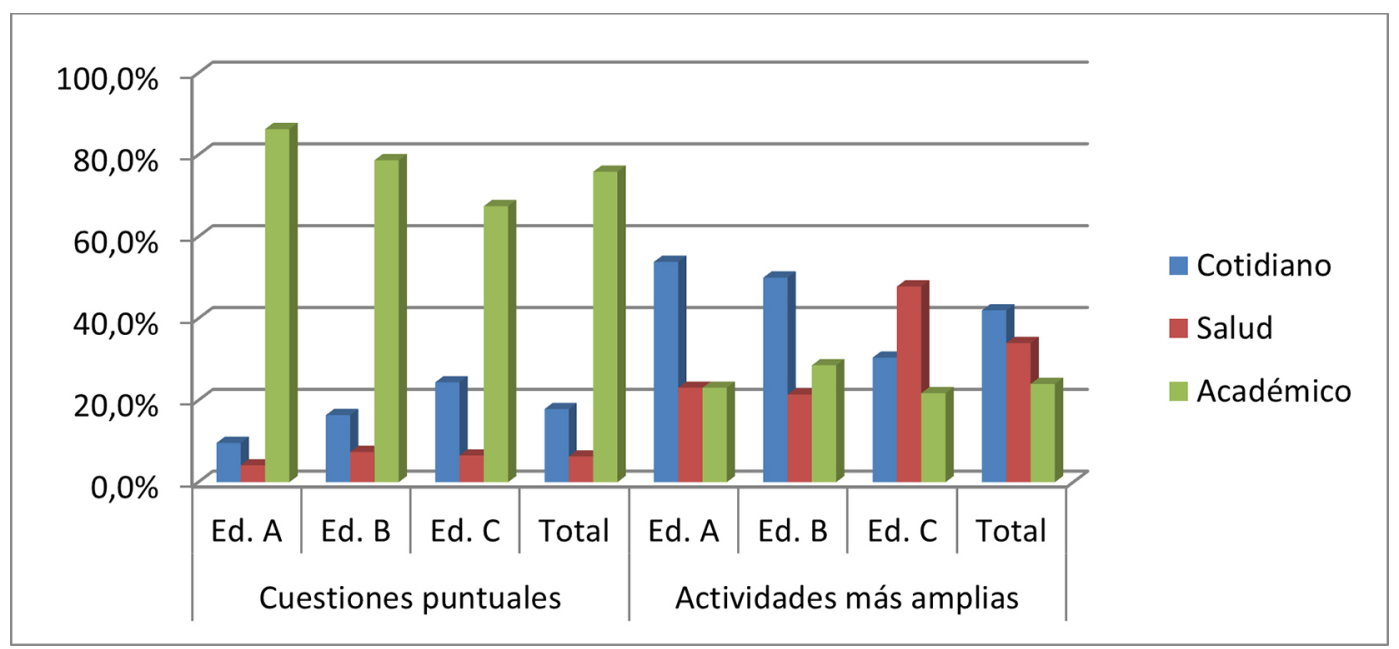

Figura 4. Distribución de las actividades según el tipo de contexto que emplean. 
Las actividades promueven el desarrollo de los diferentes tipos de capacidades de forma desigual. En cómputos totales las cuestiones puntuales atienden a una media de 1.3 tipos y las actividades más amplias a una media de 2.8. Más específicamente (Figura 5 ), las primeras se centran en capacidades tipo I, relativas al conocimiento científico en sí ( $84.4 \%$ del total) y, en menor medida, en las de tipo II, que requieren el uso de ese conocimiento en situaciones concretas (30.4\%). El porcentaje de cuestiones puntuales que implican capacidades de tipo III -uso de procedimientos científicos necesarios para abordar el estudio de situaciones-y de tipo IV -asociadas al uso de pruebas científicas- es muy reducido (11.3\% y 5.6\%). En ningún caso se aprecian diferencias entre editoriales.

La mayoría de las actividades más amplias atienden a capacidades de tipo I y II $(90.0 \%$ y $78.0 \%$, respectivamente) y algo más de la mitad contemplan capacidades de tipo III y/o IV (58.0\% y $54.0 \%$, respectivamente). Esta tendencia se aprecia también en las tres editoriales, a excepción de la editorial B, que trabaja las capacidades II y III de forma más equilibrada ( $64.3 \%$ y $71.4 \%$, respectivamente).

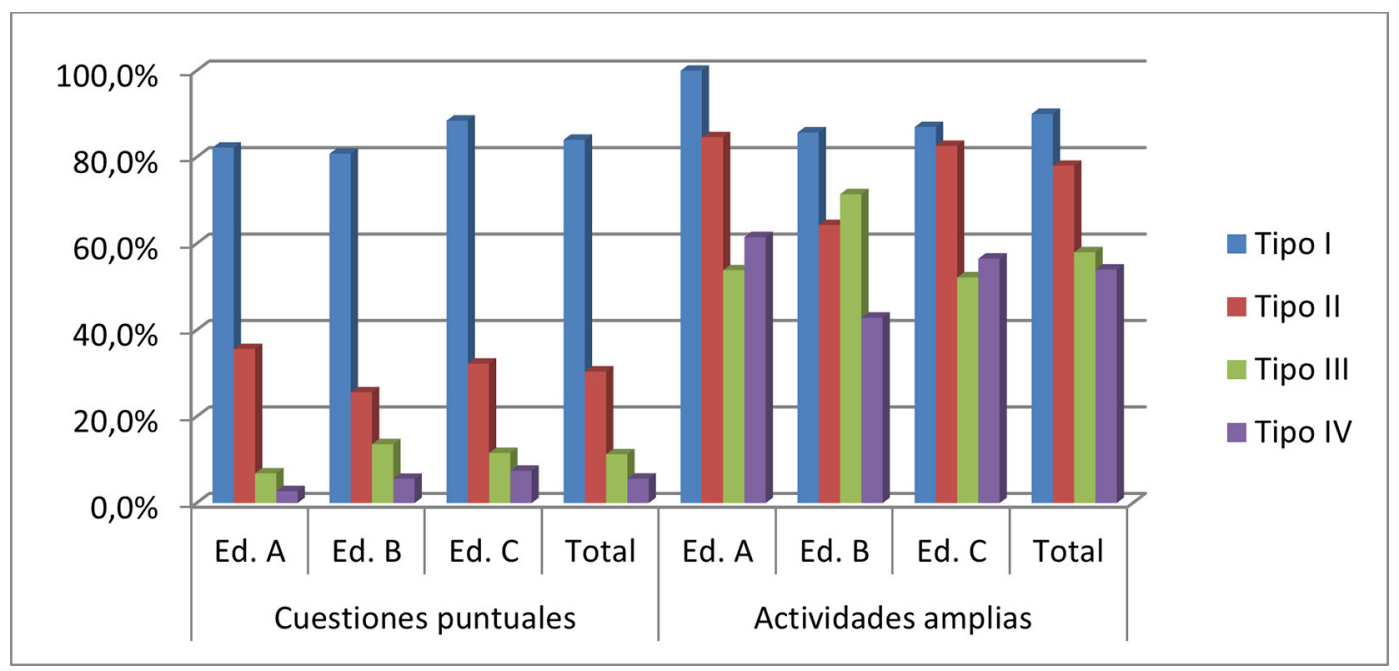

Figura 5. Proporción de actividades que trabajan los distintos tipos de capacidades

Nos centramos a continuación en las capacidades específicas que exigen las editoriales en relación con cada uno de los cuatro tipos establecidos.

En general, las actividades que trabajan capacidades de tipo I -relativas al conocimiento científico en sí- (Tabla 2), la más frecuente es la identificación de características (el 70.1\% del total de cuestiones puntuales y el $86.7 \%$ de las actividades más amplias). Además, el $44.4 \%$ de estas últimas exigen el establecimiento de relaciones y el $20 \%$ la realización de comparaciones. Sin embargo, en las cuestiones puntuales la proporción es inferior al 19\%. La definición es también una capacidad escasamente exigida en las cuestiones y prácticamente inexistente en las actividades más amplias.

En términos comparativos las actividades más amplias de las editoriales $B$ y $C$ trabajan en mayor medida que la A la identificación de características y el establecimiento de relaciones, mientras que ésta última trabaja más la comparación. En el caso de las cuestiones la editorial $C$ de nuevo trabaja más que las otras la identificación de características mientras que las editoriales A y B trabajan comparativamente más la definición. Las actividades que trabajan capacidades tipo II -relativas al uso del conocimiento en situaciones concretas- (Tabla 3) incluyen la descripción de hechos o procesos, siendo proporcionalmente más frecuentes en las actividades más amplias que en las cuestiones (69.2\% y $44.3 \%$ respectivamente). Además casi la mitad de las primeras y alrededor del $30 \%$ de las segundas exigen una expli- 
cación de cómo pasan las cosas. Sin embargo, en ambos tipos de actividades, menos del $27 \%$ exige una justificación de por qué pasan las cosas, empleando un modelo teórico de referencia. En términos comparativos, en las cuestiones puntuales la editorial A se centra en mayor medida que las otras dos en la descripción, la editorial B en la justificación y la editorial C en la explicación, existiendo menos diferencias en las actividades más amplias.

Las actividades que trabajan capacidades tipo III -uso de procedimientos científicos- (Tabla 4) incluyen sobre todo la observación y la búsqueda de información en distintas fuentes. Otros procedimientos científicos asociados al estudio de situaciones y problemas como el diseño y realización de experiencias para recoger datos tienen una presencia muy reducida en las actividades más amplias y es prácticamente inexistente en las cuestiones puntuales.

Tabla 2. Capacidades concretas que exigen las actividades que trabajan capacidades tipo I

\begin{tabular}{|l|c|c|c|c|c|c|c|c|}
\hline \multirow{2}{*}{$\begin{array}{l}\text { Capacidades } \\
\text { concretas }\end{array}$} & \multicolumn{4}{|c|}{ Cuestiones puntuales } & \multicolumn{4}{c|}{ Actividades amplias } \\
\cline { 2 - 9 } & $\begin{array}{c}\text { Ed. A } \\
\mathrm{N}=60\end{array}$ & $\begin{array}{c}\mathrm{Ed} . \mathrm{B} \\
\mathrm{N}=101\end{array}$ & $\begin{array}{c}\mathrm{Ed} . \mathrm{C} \\
\mathrm{N}=107\end{array}$ & $\begin{array}{c}\text { Total } \\
\mathrm{N}=268\end{array}$ & $\begin{array}{c}\mathrm{Ed} . \mathrm{A} \\
\mathrm{N}=13\end{array}$ & $\begin{array}{c}\text { Ed. B } \\
\mathrm{N}=12\end{array}$ & $\begin{array}{c}\text { Ed. C } \\
\mathrm{N}=20\end{array}$ & $\begin{array}{c}\text { Total } \\
\mathrm{N}=45\end{array}$ \\
\hline Identificar & 36 & 66 & 86 & 188 & 9 & 11 & 19 & 39 \\
características & $60,0 \%$ & $65,3 \%$ & $80,4 \%$ & $70,1 \%$ & $69,2 \%$ & $91,7 \%$ & $95,0 \%$ & $86,7 \%$ \\
\hline Establecer & 12 & 16 & 21 & 49 & 3 & 6 & 13 & 20 \\
relaciones & $20,0 \%$ & $15,8 \%$ & $19,6 \%$ & $18,3 \%$ & $23,1 \%$ & $50.0 \%$ & $65,0 \%$ & $44,4 \%$ \\
\hline Comparar & 5 & 10 & 18 & 33 & 4 & 2 & 3 & 9 \\
& $8,3 \%$ & $9,9 \%$ & $16,8 \%$ & $12,3 \%$ & $30,8 \%$ & $16,7 \%$ & $15,0 \%$ & $20,0 \%$ \\
\hline Definir & 14 & 20 & 5 & 39 & 1 & & & 1 \\
& $23,3 \%$ & $19,8 \%$ & $4,7 \%$ & $14,6 \%$ & $7,7 \%$ & -- & - & $2,2 \%$ \\
\hline
\end{tabular}

Nota: Los porcentajes se hallan en relación al número de actividades que exigen ese tipo de capacidad.

Tabla 3. Capacidades concretas que exigen las actividades que trabajan capacidades tipo II

\begin{tabular}{|l|c|c|c|c|c|c|c|c|}
\hline \multirow{2}{*}{$\begin{array}{l}\text { Capacidades } \\
\text { concretas }\end{array}$} & \multicolumn{4}{|c|}{ Cuestiones puntuales } & \multicolumn{4}{c|}{ Actividades amplias } \\
\cline { 2 - 9 } & $\begin{array}{c}\text { Ed. A } \\
\mathrm{N}=26\end{array}$ & $\begin{array}{c}\text { Ed. B } \\
\mathrm{N}=32\end{array}$ & $\begin{array}{c}\text { Ed. C } \\
\mathrm{N}=39\end{array}$ & $\begin{array}{c}\text { Total } \\
\mathrm{N}=97\end{array}$ & $\begin{array}{c}\text { Ed. A } \\
\mathrm{N}=11\end{array}$ & $\begin{array}{c}\text { Ed. B } \\
\mathrm{N}=9\end{array}$ & $\begin{array}{c}\text { Ed. C } \\
\mathrm{N}=19\end{array}$ & $\begin{array}{c}\text { Total } \\
\mathrm{N}=39\end{array}$ \\
\hline \multirow{2}{*}{ Describir } & 17 & 12 & 14 & 43 & 8 & 7 & 12 & 27 \\
& $65,4 \%$ & $37.5 \%$ & $35,9 \%$ & $44.3 \%$ & $72,7 \%$ & $77,8 \%$ & $63,2 \%$ & $69,2 \%$ \\
\hline \multirow{2}{*}{ Explicar } & 5 & 7 & 16 & 28 & 4 & 4 & 11 & 19 \\
\hline \multirow{2}{*}{ Justificar } & $19,2 \%$ & $21,9 \%$ & $41,0 \%$ & $28,9 \%$ & $36,4 \%$ & $44,4 \%$ & $57,9 \%$ & $48,7 \%$ \\
\hline & 4 & 13 & 9 & 26 & 3 & 1 & 4 & 8 \\
& $15,4 \%$ & $40,6 \%$ & $23,1 \%$ & $26,8 \%$ & $27,3 \%$ & $11,1 \%$ & $21,1 \%$ & $20,5 \%$ \\
\hline
\end{tabular}

Tabla 4. Capacidades concretas que exigen las actividades que trabajan capacidades tipo III

\begin{tabular}{|l|c|c|c|c|c|c|c|c|}
\hline \multirow{2}{*}{$\begin{array}{l}\text { Capacidades } \\
\text { concretas }\end{array}$} & \multicolumn{4}{|c|}{ Cuestiones puntuales } & \multicolumn{4}{c|}{ Actividades amplias } \\
\cline { 2 - 9 } & $\begin{array}{c}\text { Ed. A } \\
\mathrm{N}=5\end{array}$ & $\begin{array}{c}\mathrm{Ed} \text {. B } \\
\mathrm{N}=17\end{array}$ & $\begin{array}{c}\mathrm{Ed} . \mathrm{C} \\
\mathrm{N}=14\end{array}$ & $\begin{array}{c}\text { Total } \\
\mathrm{N}=36\end{array}$ & $\begin{array}{c}\text { Ed. A } \\
\mathrm{N}=7\end{array}$ & $\begin{array}{c}\text { Ed. B } \\
\mathrm{N}=10\end{array}$ & $\begin{array}{c}\text { Ed. C } \\
\mathrm{N}=12\end{array}$ & $\begin{array}{c}\text { Total } \\
\mathrm{N}=29\end{array}$ \\
\hline Observar & 2 & 7 & 8 & 17 & 4 & 5 & 7 & 16 \\
\hline $\begin{array}{l}\text { Buscar } \\
\text { información }\end{array}$ & 3 & 9 & 6 & 18 & 5 & 4 & 5 & 14 \\
\hline $\begin{array}{l}\text { Diseñar } \\
\text { estrategias }\end{array}$ & -- & -- & -- & -- & -- & 1 & 1 & 2 \\
\hline $\begin{array}{l}\text { Realizar } \\
\text { experiencias }\end{array}$ & 1 & 1 & -- & 2 & 2 & 1 & 1 & 4 \\
\hline
\end{tabular}


Finalmente, las actividades que exigen capacidades tipo IV -el uso de pruebas- (Tabla 5) se centran en el análisis de información. Sin embargo, la elaboración de conclusiones a partir de las pruebas obtenidas y la argumentación, tienen una presencia más reducida tanto en las cuestiones puntuales como en las actividades más amplias.

Tabla 5. Capacidades concretas que exigen las actividades que trabajan capacidades tipo IV

\begin{tabular}{|l|c|c|c|c|c|c|c|c|}
\hline \multirow{2}{*}{ Capacidades concretas } & \multicolumn{4}{|c|}{ Cuestiones puntuales } & \multicolumn{4}{c|}{ Actividades amplias } \\
\cline { 2 - 10 } & $\begin{array}{c}\text { Ed. A } \\
\mathrm{N}=2\end{array}$ & $\begin{array}{c}\text { Ed. B } \\
\mathrm{N}=7\end{array}$ & $\begin{array}{c}\text { Ed. C } \\
\mathrm{N}=9\end{array}$ & $\begin{array}{c}\text { Total } \\
\mathrm{N}=18\end{array}$ & $\begin{array}{c}\text { Ed. A } \\
\mathrm{N}=8\end{array}$ & $\begin{array}{c}\text { Ed. B } \\
\mathrm{N}=6\end{array}$ & $\begin{array}{c}\text { Ed. C } \\
\mathrm{N}=13\end{array}$ & $\begin{array}{c}\text { Total } \\
\mathrm{N}=27\end{array}$ \\
\hline Analizar información & 2 & 4 & 8 & 14 & 6 & 6 & 10 & 22 \\
\hline Elaborar conclusiones & -- & 2 & -- & 2 & 2 & 1 & 4 & 7 \\
\hline Argumentar & -- & 1 & 1 & 2 & 2 & 1 & 2 & 5 \\
\hline
\end{tabular}

\section{Conclusiones. Discusión}

En este trabajo se pretendía analizar la tipología de las actividades que plantean los libros de texto de 6ㅇ de Educación Primaria, en las editoriales más empleadas por el profesorado y en qué medida las actividades analizadas promueven el desarrollo de capacidades asociadas a la competencia científica de los estudiantes

Respecto a la tipología de las actividades:

- La mayoría son cuestiones puntuales, que se sitúan intercaladas o al final de cada unidad didáctica y que, en general, no incluyen ningún recurso específico para la obtención de información respecto a la cuestión planteada. Además, la mayor parte de ellas se proponen en un contexto académico.

- Las actividades más amplias se sitúan de forma intercalada o al final pero también al inicio de la unidad didáctica, la mayoría incluyen algún recurso específico para la obtención de información y se proponen en un contexto cotidiano o de salud.

Respecto al tipo de capacidades que trabajan:

- Las cuestiones puntuales se centran en capacidades tipo I, relacionadas con el aprendizaje de hechos y conceptos científicos, siendo la más frecuente la identificación de características o partes de objetos o procesos.

- Las actividades más amplias, además de las capacidades anteriores, exigen el uso del conocimiento disponible para describir o explicar situaciones específicas. Sin embargo, la justificación se solicita en un reducido número de actividades.

- Ambos tipos de actividades prestan poca atención al uso de procedimientos científicos necesarios para abordar el estudio de situaciones, como el diseño y realización de experiencias, así como al uso de pruebas para elaborar conclusiones y argumentaciones. .

Lo indicado nos conduce a pensar que la mayoría de las actividades que incluyen los libros habitualmente empleados en las aulas de Primaria no son muy diferentes a las clásicas cuestiones que planteaban los textos hace años. Así, las actuales cuestiones siguen siendo meras tareas atomizadas que se presentan al alumnado como refuerzo del conocimiento teórico previamente expuesto en el propio texto, a pesar de que este tipo de planteamientos han sido ampliamente criticados en cuanto a su escasa rentabilidad educativa (Martínez Losada y García Barros, 2003). Además, aunque existe un importante consenso en cuanto a la importancia de abordar el estudio de situaciones próximas a los niños y niñas de Primaria (Gilbert, 2006, Sanmartí y otros, 2011), el tipo de cuestiones que proponen 
los textos es muy academicista, por lo que, aunque podrían ser relevantes desde el punto de vista científico, resultan poco significativas y funcionales para el alumnado. Todo ello es coherente, a su vez, con el énfasis que se hace en el uso de ciertas habilidades directamente relacionadas con el conocimiento científico en sí, en detrimento de otras que en el marco competencial actual se consideran imprescindibles (Pro, 2013). Asimismo, el hecho de que las actividades incidan sobre todo en aquellas habilidades poco exigentes desde el punto de vista cognitivo, como por ejemplo la mera identificación de características, en detrimento de otras como el establecimiento de relaciones o la definición, hace que no se esté incidiendo suficientemente en la finalidad última de este tipo de habilidades, que no es otro que la adquisición de conocimiento científico en sí mismo.

Por otra parte, si bien en las actividades más amplias parece existir un cierto avance, tanto en lo que se refiere a la conexión con hechos y situaciones del entorno de los escolares, como al uso de ciertas capacidades implicadas en la competencia científica, lo cierto es que son poco frecuentes en los textos habituales. Dicho avance es además relativo y claramente insuficiente pues siguen poniendo énfasis en las capacidades concretas menos exigentes desde el punto de vista competencial, como por ejemplo la descripción de hechos, la observación/ búsqueda de información puntal sobre ellos o el análisis de los mismos, frente a su justificación teórica, la propuesta de estrategias o la elaboración de conclusiones argumentadas.

Lo anteriormente señalado pone de manifiesto la resistencia al cambio de los textos escolares y la necesidad de mejora del tipo y planteamiento de las actividades que incluyen. No podemos olvidar que, a pesar de que el profesorado es el último responsable del tipo y orientación de las actividades que se llevan a cabo en el aula, y a pesar de que puedan emplear también otros materiales, los libros de texto son un referente importante de las decisiones docentes del profesorado (Occelli y Valeiras, 2013). Por tanto, podrían ser también un elemento clave en la innovación de la enseñanza, siempre y cuando superasen la preponderancia de actividades atomizadas, dirigidas a reproducir el conocimiento, lo que requiere el uso de capacidades cognitivas poco exigentes. Para ello, será necesario priorizar actividades que favorezcan la capacidad de emplear ese conocimiento en contextos concretos donde las cuestiones y problemas resulten significativos, que den ocasión a emplear En este marco a lo largo de la Educación Primaria se podrá potenciar el uso de capacidades más variadas y progresivamente más exigentes, imprescindibles para el desarrollo de la competencia científica de la ciudadanía actual.

\section{Agradecimientos}

Trabajo realizado en el marco del proyecto EDU2016-79563-R.

\section{Referencias bibliográficas}

Blanco, A. y España. E. (2012). Contexto y enseñanza de la competencia científica. Alambique, 70, 9-18.

Blanco López, A., España Ramos, E. y Franco-Mariscal, A.J. (2017). Estrategias didácticas para el desarrollo del pensamiento crítico en el aula de ciencias. Ápice. Revista de Educación Científica, 1(1), 107-115. DOI: https://doi.org/10.17979/ arec.2017.1.1.2004

Blanco-López, A., España-Ramos, E., González-García, F.J. y Franco-Mariscal, A.J. (2015). Key Aspects of Scientific Competence for Citizenship: A Delphi Study of the Expert Community in Spain. Journal of Research in Science Teaching 52 (2): 164-198. DOI: https://doi.org/10.1002/tea.21188 
Cañal, P. (2011). Competencia científica y competencia profesional en la enseñanza de la biología y la geología. En P. Cañal (Ed.), Didáctica de la Biología y la Geología. Barcelona: Ministerio de Educación. Graó.

Cañal, P. (2012). La evaluación de la competencia científica requiere nuevas formas de evaluar los aprendizajes. En E. Pedrinaci (Ed.), Once ideas clave El desarrollo de la competencia Científica. Barcelona: Graó.

Cañas, A., Martín-Díaz, M. y Nieda, J. (2007). Competencia en el conocimiento y la interacción con el mundo físico. La competencia científica. Madrid: Alianza Editorial.

Cañas, A. y Martín-Díaz, M. (2010). ¿Puede la competencia científica acercar la ciencia a los intereses del alumnado? Alambique, 66, 80-87.

COSCE-Confederación de Sociedades Científicas de España (2011). Informe ENCIENDE. Enseñanza de las Ciencias en la Didáctica Escolar para edades tempranas en España. Madrid: COSCE. Recuperado de: http://www.cosce.org/disponible-el-informeenciende/

Del Carmen, L. (2001). Los materiales de desarrollo curricular: un cambio imprescindible. Investigación en la Escuela, 43, 51-56.

Díaz-Moreno, N. y Jiménez-Liso, R. (2012). Las controversias sociocientíficas: temáticas e importancia para la educación científica. Revista Eureka sobre Enseñanza y Divulgación de las Ciencias, 9(1), 54-70. Recuperado de: http://hdl.handle. net/10498/14624

Fensham, P. (2009). Real world contexts in PISA Science: Implications for context-based science education. Journal or Research in Science Teaching, 46(8), 884-896. DOI: https://doi.org/10.1002/tea.20334

Gilbert, J.K. (2006). On the nature of context in chemical education. International Journal of Science Educactión, 28(9), 957-976. DOI: https://doi. org/10.1080/09500690600702470

González Rodríguez, C., Martínez Losada, C. y García Barros, S. (2009). Las programaciones de ciencias en secundaria. ¿Un documento útil o simplemente burocrático? Revista Electrónica de Enseñanza de las Ciencias, 8(3), 1079-1093.

Gutiérrez, A. (2006). PISA y la evaluación de la alfabetización científica. Investigación en la Escuela, 60, 65-77.

Kauertz, A., Neumann, K. y Haertig, H. (2012). Competence in Science Education. Second International Handbook of Science Education. Springer Netherlands, 711-721.

López, A. y Postigo, Y. (2014). Análisis de las imágenes del cuerpo humano en libros de texto españoles de primaria. Enseñanza de las Ciencias: revista de investigación y experiencias didácticas, 32(3), 551-570. DOI: https://doi.org/10.5565/rev/ ensciencias.1319

Martínez Losada, C. y García Barros, S. (2003). Las actividades de Primaria y ESO incluidas en libros escolares ¿Qué objetivos persiguen? ¿Qué procedimientos enseñan? Enseñanza de las Ciencias, 21(2), 243-264.

MECD (2016). PISA 2015. Programa para la Evaluación Internacional de los Alumnos. Informe español. Madrid: MEC. Recuperado de: http://www.educacionyfp.gob.es/ inee/dam/jcr:e4224d22-f7ac-41ff-a0cf-876ee5d9114f/pisa2015preliminarok.pdf 
Occelli, M., y Valeiras, N. (2013). Los libros de texto de ciencias como objeto de investigación: una revisión bibliográfica. Enseñanza de las Ciencias, 31(2), 133-152.

OECD (2002). Definition and Selection of Competencies: Theoretical and Conceptual Foundations. Summary of the final report "Key Competencies for a Successful Life and a Well-Functioning Society". Recuperado de: http://www.portalstat.admin.ch/deseco/deseco_finalreport_summary.pdf

Osborne, J. (2014). Teaching scientific Practices: Meeting the Challenge of Change. Journal of Science Teacher Education, 25(2), 177-196.

Pedrinaci, E. (2012). El ejercicio de una ciudadanía responsable exige disponer de cierta competencia científica. En: E. Pedrinaci (coord.). 11 ideas clave. El desarrollo de la competencia científica. Barcelona: Graó.

Pro Bueno, A. (2013). Enseñar procedimientos: por qué y para qué. Alambique, 73, 69-76

Rocard, Y. (2007) Science Education Now. Report EU22-845, European Commission, Brussels. Disponible on-line en: http://ec.europa.eu/research/science-society/ document_library/pdf_06/report-rocard-on-science-education_en.pdf

Rodríguez, F., de las Heras, M. A., Romero, R. y Cañal, P. (2014). El conocimiento escolar sobre los animales y las plantas en primaria: Un análisis del contenido específico en los libros de texto. Revista Electrónica de Enseñanza de las Ciencias, 13(1), 97-114. Recuperado de: http://reec.uvigo.es/volumenes/volumen13/REEC_13_1_6_ex761. pdf

Rodríguez, J., Pro, A. y Molina, M.D. (2018). Opinión de los docentes sobre el tratamiento de las competencias en los libros de texto de Ciencias de la Naturaleza en Educación Primaria. Revista Eureka sobre Enseñanza y Divulgación de las Ciencias, 15(3), 3102. DOI: http://dx.doi.org/10.25267/Rev_Eureka_ensen_divulg_cienc.2018.v15. i3.3102

Romero-Ariza, M. (2017). El aprendizaje por indagación, ¿existen suficientes evidencias sobre sus beneficios en la enseñanza de las ciencias? Revista Eureka sobre Enseñanza y Divulgación de las Ciencias 14 (2), 286-299. DOI: http://dx.doi.org/10.25267/ Rev_Eureka_ensen_divulg_cienc.2017.v14.i2.01

Sanmartí, N. y Márquez, C. (2017). Aprendizaje de las ciencias basado en proyectos: del contexto a la acción. Ápice. Revista de Educación Científica, 1(1), 3-16. DOI: https:// doi.org/10.17979/arec.2017.1.1.2020

Sanmartí, N. y Sardá, A. (2007). El caso PISA. Cuadernos de Pedagogía, 370, 60-63.

Sanmartí, N., Burgoa, B. y Nuño, T. (2011). ¿Por qué el alumnado tiene dificultad para utilizar sus conocimientos científicos escolares en situaciones cotidianas? Alambique, 67, 62-69.

Vilches, A. y Gil, D. (2010). El programa PISA: un instrumento para la mejora del proceso de enseñanza-aprendizaje. Revista Iberoamericana de Educación, 53, 121-154. 
